\title{
Arrival direction reconstruction of ultra-high-energy neutrinos with ARA
}

\author{
Myoungchul Kim*, Simon Archambault, Keiichi Mase, Shigeru Yoshida ${ }^{\dagger}$, Aya \\ Ishihara for the ARA Collaboration \\ Chiba University \\ E-mail: mkimeicecube.wisc.edu
}

\begin{abstract}
Askaryan Radio Array (ARA) deploys antennas in the Antarctic ice with a 3D lattice shape, looking for Askaryan emission produced by interactions of high-energy neutrinos. Currently, the construction of the detector is ongoing, and five independent stations have been deployed. Two types of antennas are used to measure the polarization of the radio wave produced by neutrino-induced showers. Each antenna is designed to be sensitive to a vertically or horizontally-polarized radio wave. The polarization information is important for reconstructing the neutrino direction. We developed a method to reconstruct the neutrino arrival direction by calculating the polarization of the signal using information of two types of antennas. We present the reconstruction method and the angle difference of a true simulation angle and reconstructed angle with taking into account the vertex and detector uncertainties. A bulk ice with a constant refractive index is used in the study. The more realistic angular resolution of neutrino arrival direction will be evaluated with the realistic South Pole ice model in future.
\end{abstract}

36th International Cosmic Ray Conference -ICRC2019-

July 24th - August 1st, 2019

Madison, WI, U.S.A.

\footnotetext{
${ }^{*}$ Corresponding author

$\dagger$ Speaker.

$\ddagger$ ARA author list available at http://ara.wipac.wisc.edu/authorlist.
} 


\section{Introduction}

Ultra-High Energy Cosmic Rays (UHECRs) have been measured by the giant telescopes such as Telescope Array (TA) and Pierre Auger Observatory [1,2]. The origin of the UHECRs is still mystery. UHE neutrinos are expected to be produced when UHECRs interact with Cosmic Microwave Background (CMB) photons $[3,4,5]$. Thus, UHE neutrinos can reveal the origin of UHECRs. Also, due to the nature of the neutrino as a charge-less and weakly interacting particle, it rarely interacts with matter. Therefore, a neutrino becomes an important particle for investigating the deep universe and studying powerful accelerators.

Detecting the cosmogenic neutrinos are challenging. The low cross-section of a neutrino interaction with matter leads to the event rate of $\sim 1$ event per gigaton per year for UHE neutrinos [6]. This makes the ice in Antarctica, a large volume of radio transparent medium with a long radiowave attenuation length of $\sim 1 \mathrm{~km}$ [7], optimal for constructing a detector of the size necessary for neutrino astronomy.

UHE neutrinos interact with the Antarctic ice to produce electromagnetic cascades. When the wavelength of the Cherenkov radiation is longer than the shower size, which is about $10 \mathrm{~cm}$, the emitted radiation will be coherent due to the so-called Askaryan effect $[8,9]$. These coherent radio waves do not scatter, in contrast to the optical light; thus radio should be able to give us an improved angular resolution for the neutrino direction in theory. Therefore, studying these coherent radio waves can provide a precise angular resolution, which is favorable for searching the sources of UHECRs. This proceeding focuses on the reconstruction of the neutrino direction by observing the polarization of the radio signals by the two types of antennas in ARA.

\section{The ARA Detector}

The Askaryan Radio Array (ARA) is the largest neutrino detector under construction near the geographic South Pole in the ice. It is specifically designed to utilize the Askaryan effect to detect high energy neutrinos above $10^{16} \mathrm{eV}$ [10]. Currently, five independent stations separated by $2 \mathrm{~km}$ spacing have been deployed. Each station consists of $16 \mathrm{RF}$ antennas equipped with 4 downhole strings in the $200 \mathrm{~m}$ below the surface, and 4 calibration pulsers on 2 strings have been deployed $\sim 40 \mathrm{~m}$ horizontally away from the station, as shown in Fig. 1. The data acquisition (DAQ), placed at the center of the station, digitizes and transmits the signal to the main server [11].

The lattice shape, with $30 \mathrm{~m}$-long baselines, is valuable for vertex reconstruction using time information. Two types of antennas, vertically-polarized (Vpol) and horizontally-polarized (Hpol), are deployed to measure the polarization of the electric field (E-field) in order to reconstruct the incoming neutrino direction.

\section{Reconstruction of Neutrino Direction}

\subsection{E-field of The Cherenkov Radiation}

The coherence of the Askaryan radiation gives us a stronger Cherenkov signal than optical. By following theoretical work by E. Zas, F. Halzen, T. Stanev and J. Alvarez [8, 9], we decided to use two major characteristics of E-field for the neutrino direction. 


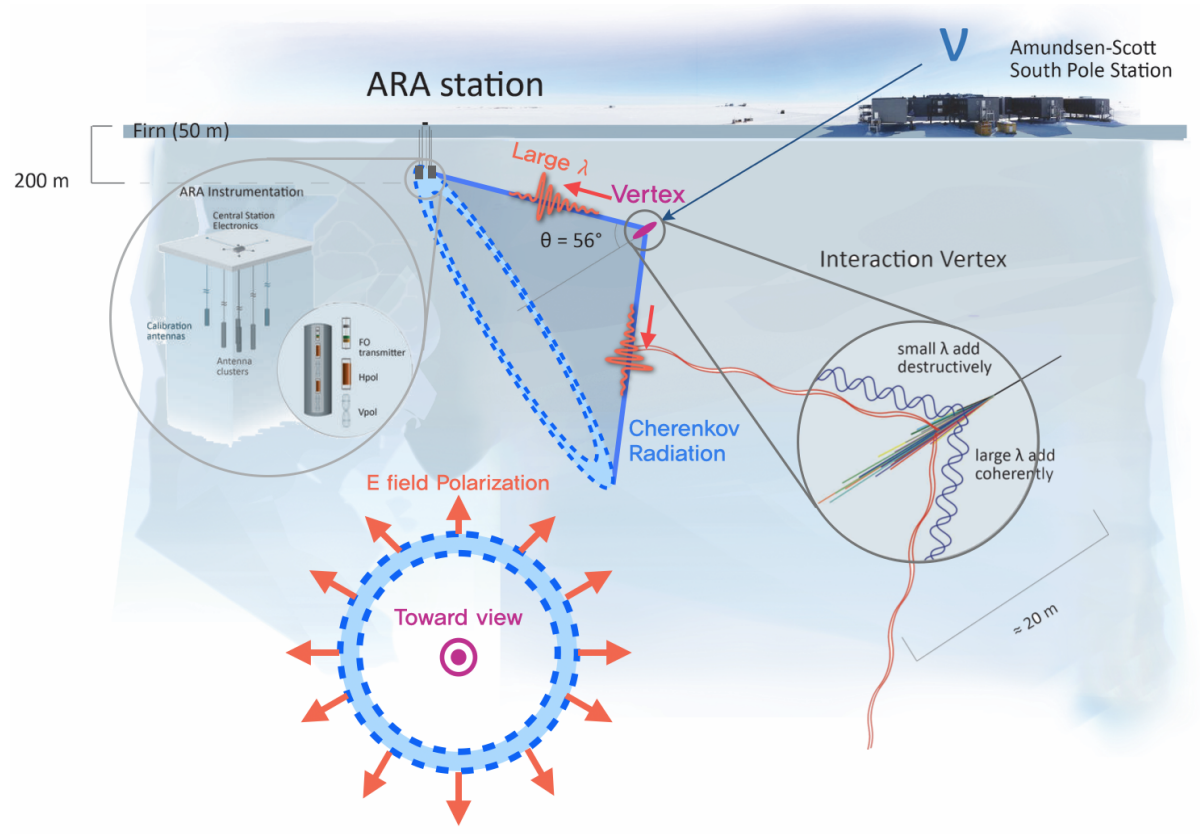

Figure 1: Design of the ARA detector. When a neutrino interacts with the ice, a coherent radio pulse is generated. The clock face of the Cherenkov cone has a different polarization of the E-field. Vpol and Hpol antennas record the radio signal. The signal is amplified by signal chain electronics.

The polarization vector of the E-field is perpendicular to the direction of a radio wave [8]. The direction of a radio signal at the Cherenkov angle is $56^{\circ}$ from a shower axis in deep ice, which aligns with the incoming neutrino direction [9]. Therefore, a direction of the E-field polarization depends on where the antenna is placed on the cone's "clock face". Detection of this polarized Efield by the antenna will point to a specific part of the cone, which can be used to infer the neutrino direction.

In the observed E-field frequency spectrum, a cut-off behavior depends on the viewing angle with respect to the Cherenkov cone. This cut-off in the spectrum appears at a frequency where the coherence of the E-field breaks down [8]. Since the ARA antennas are broadband quantify, they can resolve where the frequency contribution from the Askaryan radiation ends [9]. Comparing spectral cutoffs in 16 antennas in a station can indicate how far the Cherenkov cone is from the detector and increase the accuracy of the neutrino direction reconstruction.

In the ARA detector at South Pole in the ice, The E-field vectors are measured in the vertical and horizontal projection, limiting the observation of E-field vector to the $\mathrm{Z}$ component with Vpol antennas and the component in the XY plane with Hpol antennas. We denote $\mathbf{E}_{\mathbf{v}}$ and $\mathbf{E}_{\mathbf{h}}$ as the observed E-field vectors in the Vpol and Hpol channels, respectively.

\subsection{Stokes Parameters}

In order to calculate the polarization angle of the E-field, we introduce Stokes parameters. It is convenient to describe the polarization angle of the EM radiation. Considering $\mathbf{E}_{\mathbf{v}}$ and $\mathbf{E}_{\mathbf{h}}$ as the two-vector components of the E-field, we can derive two Stokes parameters given by Eq. 3.1 
and 3.2. From the Stokes parameters, $U$ and $Q$, polarization angle, $\theta_{E}$ can be expressed as in Eq. 3.3. [12]

$$
\begin{aligned}
Q & =\left|\mathbf{E}_{\mathbf{v}}\right|^{2}-\left|\mathbf{E}_{\mathbf{h}}\right|^{2} \\
U & =2 \operatorname{Re}\left(\mathbf{E}_{\mathbf{v}} \mathbf{E}_{\mathbf{h}}{ }^{*}\right) \\
\theta_{E} & =\frac{1}{2} \tan ^{-1}\left(\frac{U}{Q}\right)
\end{aligned}
$$

Since the Cherenkov radiation is emitted in a cone-shape as in Fig. 1, E-field observed at each angle around the clock face of the cone will have a unique polarization angle. The reconstructed polarization angle of E-field obtained from Eq. 3.3 indicates at which angle around the Cherenkov cone the radiation is being observed.

In order to reconstruct the 3D angular distribution of the Cherenkov radiation forming a ring from observation, knowing the interaction position is a necessary [11]. Finally, considering the Cherenkov angle $\left(56^{\circ}\right)$ in deep ice between the radiation and neutrino direction, reconstructing the radiation in $3 \mathrm{D}$ results in an estimation of the incoming neutrino nadir $(\theta)$ and azimuth $(\phi)$. Fig. 2 is a schematic of demonstrating the transformation of a set of polarization angles to neutrino direction. In reality, uncertainty from reconstructed vertex region and uncertainty of detector response will affect neutrino direction. In this proceeding, the study was performed with simulation take into account those uncertainties.

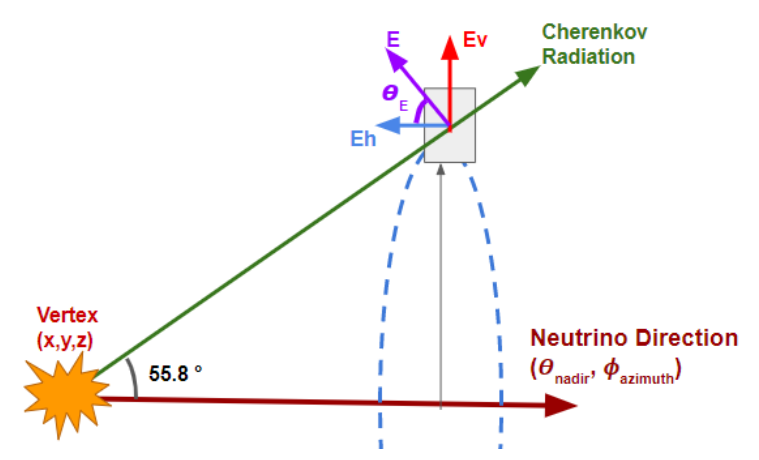

Figure 2: Schematic of neutrino direction reconstruction. Once we reconstruct the polarization angle, $\theta_{E}$, neutrino direction will be calculated with the vertex position.

\section{Analysis}

\subsection{Simulation}

Although the diffuse neutrino search is ongoing with the latest data [13], ARA has not found a high energy neutrino in the recorded data [11]. Therefore, this study was performed with simulation. The simulation we used in this study is called AraSim [14]. It generates thermal noise background expected from the South pole ice, and has an Askaryan radiation model based on a work by Alvarez-Muniz et. al [9]. Simulation takes care of the signal generation, propagation in 
ice and the detector response including the antenna response. Also, neutrino flavor, interaction type (EM or hadronic shower), and neutrino energy can be user configured. Due to the characteristic of the South pole ice, the depth-dependent index of refraction is also implemented but in the current study, it is turned off [15]. It will be applied in future. The simulation also models the two types of antennas based on an in-situ measurement. The trigger logic is also same as the real detector configuration.

In the simulation, fluctuated vertex positions to reflect the uncertainties of the vertex resolution $\left(0.5^{\circ}\right)$ and distance-to-vetex direction. Then, we generated Cherenkov radiations for different neutrino directions $(50 \%)$.

Fig. 3 shows example geometries for three different polarization angles. We denote $0^{\circ}$ and $180^{\circ}$ as the angles where the polarization is horizontal. $90^{\circ}$ is when the polarization is vertical. Polarizations in the $0 \sim 180^{\circ}$, range are simulated at $15^{\circ}$ intervals. In this simulation, ray paths were constrained to always hit the one antenna among 16 antennas in a station.
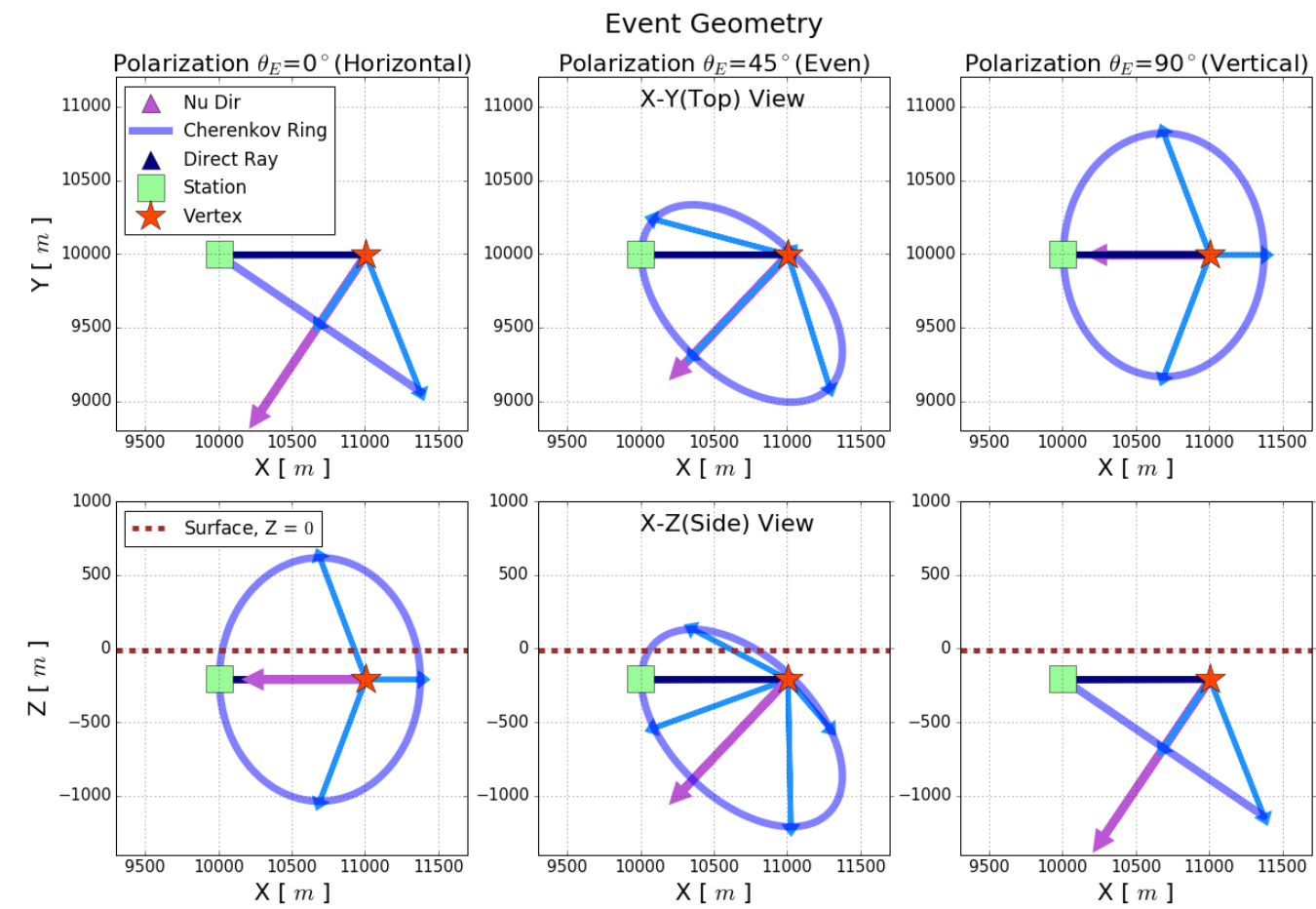

Figure 3: The geometry of 3 neutrino events. Each event is expressed in $X-Y$ and $X-Z$ plane. The interaction position is identical, different neutrino incoming direction and Cherenkov radiation are illustrated based on polarization angle setting $\left(0^{\circ}, 45^{\circ}\right.$ and $\left.90^{\circ}\right)$.

The energy range of signals considered is $10^{18 \sim 20} \mathrm{eV}$ and only an electromagnetic (EM) shower was generated. Thermal noise was included in every event. The noise power level follows that expected from the $325 \mathrm{~K}$ system temperature, which is the sum of the ambient environmental temperature and the system thermal noise. At each frequency, a different phase was applied to the noise when transforming it from the frequency domain to the time domain. Then, the noise is added to the Askaryan emission. Fig. 4 shows an example simulated waveform read out by the detector at $10^{18} \mathrm{eV}$, at polarization angles of $0^{\circ}, 45^{\circ}$ and $90^{\circ}$. 


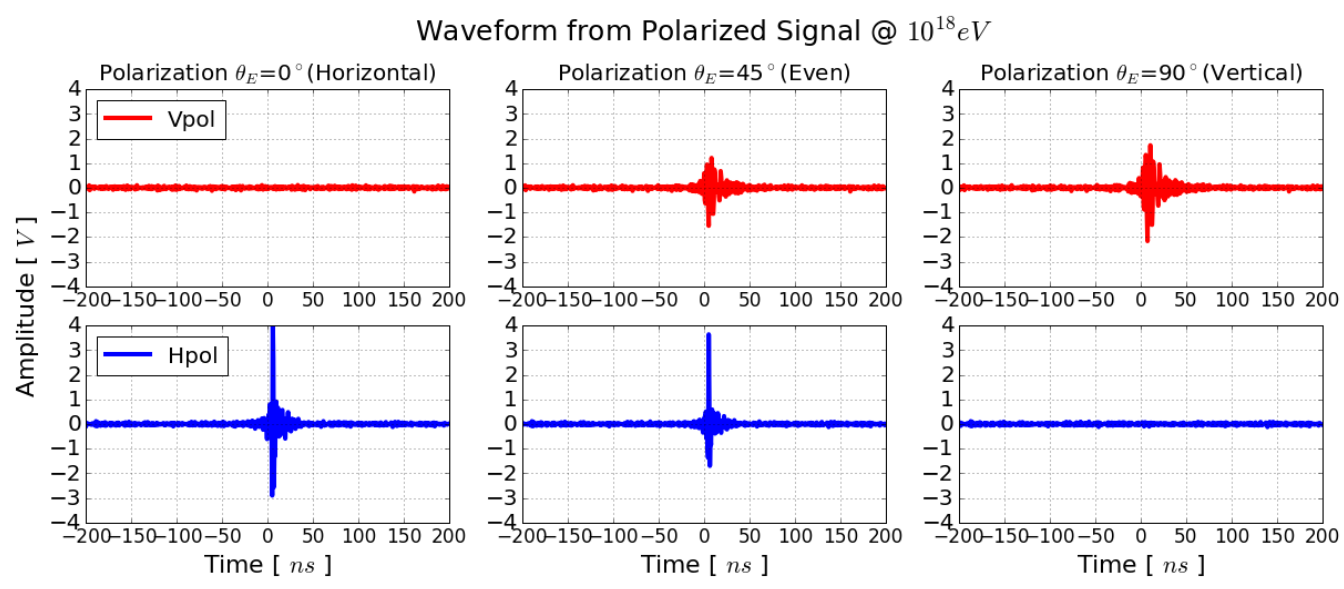

Figure 4: Readout waveform of three simulated neutrino events. Simulation settings are the same as Fig. 3. Behaviors of the waveform are seen for three different polarization settings.

\subsection{E-field Reconstruction}

In order to reconstruct the E-field from the recorded data, the detector response must be precisely understood. The detector response is separated into the antenna responses and the response of the detector's signal process chain. Since the antenna response has a zenith angle dependence, the corresponding angle was calculated using the last step of the ray vector before reaching the antenna. The signal chain consists of an Low Noise Amplifier (LNA), a band-pass filter and Radio Frequency over Fiber (RFoF). The LNA increases the strength of the signal before sending it to the DAQ. The gain of the signal chain depends on frequency but does not depend on the direction of the signal.

The readout waveform was transformed into a frequency spectrum by following a Fast Fourier Transform. All of the detector response was removed in the frequency domain. After removing the detector response, the resulting signal is a combination of E-field and noise in the frequency spectrum. Fig. 5 is the reconstructed E-field spectra of the waveforms in Fig. 4. The plots superpose the reconstructed E-field and simulated true input signal without noise. For polarization angles of $0^{\circ}, 90^{\circ}$ and $180^{\circ}$, the signal of the less dominant polarization is smaller than the thermal noise. Therefore, the reconstructed signal is more affected by noise.

After summing up the frequency spectrum of E-field, we fluctuated each 16 E-field based on systematic uncertainty of the detector. The systematic uncertainty was calculated from difference between in-situ measurement and the obtained antenna model. 19\% (Vpol) and 16\% (Hpol) of uncertainties were used for fluctuating E-field.

\subsection{Systematic Uncertainties}

Polarization angle calculations are based on the reconstructed E-field spectra using Stokes parameters [12]. The E-field of pair of neighboring antennas, which are Vpol and Hpol, are used for $\mathbf{E}_{\mathbf{v}}$ and $\mathbf{E}_{\mathbf{h}}$. A total of 8 antenna pairs are used for reconstructing polarization angles. Eq. 3.3 suggests that the reconstructed angle is heavily dependent on the ratio of E-fields measured in an antenna pair. In order to increase the accuracy of the reconstruction, we introduced two types of 


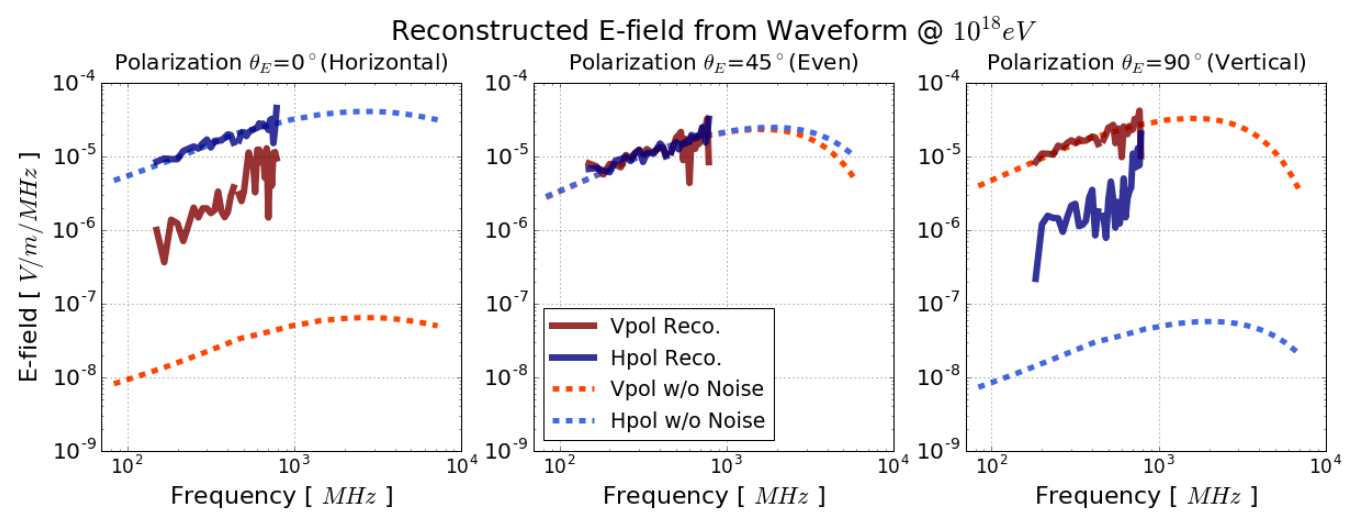

Figure 5: Reconstructed E-field from Fig. 4. Vpol and Hpol antenna results are plotted in the same figure. The dashed line is the simulated input E-field without noise in. The solid line is the reconstructed E-field.

frequency cuts in E-field spectrum. First, based on in-situ antenna gain profile, we decided to use the E-field spectrum below $400 \mathrm{MHz}$. And second, when the reconstructed E-field amplitude gets within the thermal noise level, the frequency cut was applied.

Using the reconstructed polarization angles taking into account vertex and detector uncertainties, neutrino directions are reconstructed as a nadir and azimuth angle. Fig. 6 is a comparison between the reconstructed value and true for different energies and polarization angles. We find that we can reconstruct the neutrino arrival direction with an accuracy of about $6^{\circ}$ in case of the simple bulk ice. We found the difference mostly comes from the vertex and the detector uncertainties. We are going to investigate the effect of the ice model and the uncertainty of the model in future to obtain the realistic angular resolution.

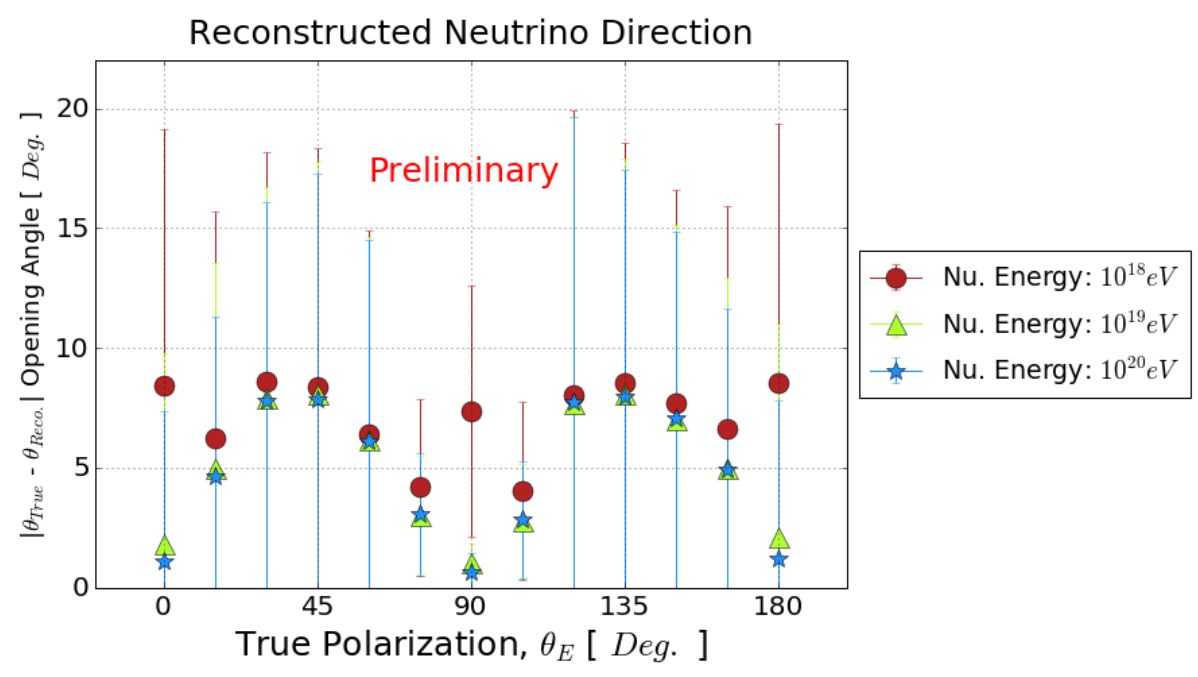

Figure 6: Systematic angle difference of reconstructed neutrino direction and the true direction. Each data point is an average of 8 antenna pairs. The error bar represents the standard deviation. 


\section{Summary and Outlook}

We present a method for reconstructing neutrino directions by leveraging the polarization information recorded in the ARA detector. By unfolding the detector response from simulated events that included thermal noise, we were available to reconstruct the expected E-field from neutrinos to with an accuracy of about $6^{\circ}$. The uncertainties of the vertex and the detectors are taken into account, but we assume a simple bulk ice with a constant refractive index for the ice. We will investigate the effect of the ice model in future to finalize the results. We also found that the systematic angle difference of the true angle and the reconstructed angle mostly comes from the uncertainties of the vertex and the detector. Therefore, we need to improve the vertex position reconstruction and to understand our detector to improve the neutrino directional reconstruction for the neutrino astronomy.

\section{References}

[1] G.R. Farrar, The Astrophysical Journal 623, 164âĂŞ170 (2005).

[2] The Pierre Auger Collaboration, ApJ 802, 111 (2015)

[3] K. Greisen, Phys. Rev. Lett. 16, 748-750 (1966).

[4] G. Zatsepin and V. Kuzmin, JETP Lett. 4, 78 (1966).

[5] V. Beresinsky and G. Zatsepin, Phys. Lett. B. 28, 423-424 (1969).

[6] R. Gandhi, C. Quigg, M. H. Reno, and I. Sarcevic, Astroparticle Physics 5, 81 (1996).

[7] P. Allison et al. (ARA), Astropart. Phys. 35457 (2012).

[8] E. Zas, F. Halzen, and T. Stanev, Phys. Rev. D 45, 362 (1992).

[9] J. Alvarez-Muiz and E. Zas, Physics Letters B 411, 218 (1997).

[10] G. A. Askaryan, JETP 14, 441 (1962).

[11] P. Allison et al. (ARA), Phys. Rev. D 93082003 (2016).

[12] Stokes, G. G. (1852). On the composition and resolution of streams of polarized light from different sources. Transactions of the Cambridge Philosophical Society, 9, 399.

[13] Ming-Yuan Lu et al. (ARA), in proceedings of the 35th ICRC (2017).

[14] P. Allison et al. (ARA), Astropart. Phys. 70, 62 (2015).

[15] I. Kravchenko, D. Besson, J. Meyers, Journal of Glaciology 50 522-532 (2004). 